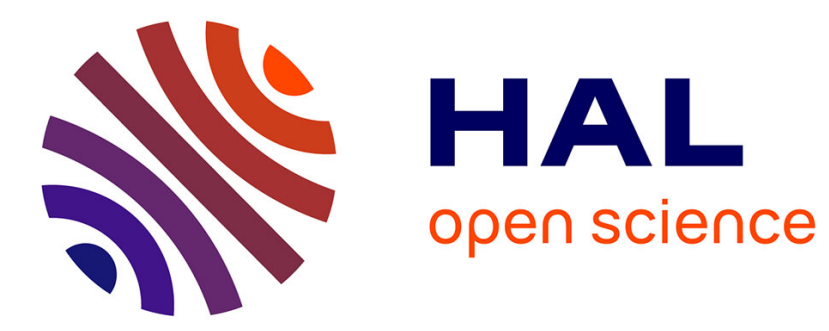

\title{
Dyson's equations for the (Ising) spin-glass
}

Cirano de Dominicis, I. Kondor, T. Temesvari

\section{To cite this version:}

Cirano de Dominicis, I. Kondor, T. Temesvari. Dyson's equations for the (Ising) spin-glass. Journal de Physique I, 1994, 4 (9), pp.1287-1308. 10.1051/jp1:1994189 jpa-00246992

\section{HAL Id: jpa-00246992 https://hal.science/jpa-00246992}

Submitted on 1 Jan 1994

HAL is a multi-disciplinary open access archive for the deposit and dissemination of scientific research documents, whether they are published or not. The documents may come from teaching and research institutions in France or abroad, or from public or private research centers.
L'archive ouverte pluridisciplinaire HAL, est destinée au dépôt et à la diffusion de documents scientifiques de niveau recherche, publiés ou non, émanant des établissements d'enseignement et de recherche français ou étrangers, des laboratoires publics ou privés. 
Classification

Physics Abstracts

$05.20-75.10 \mathrm{~N}-75.50 \mathrm{~L}$

\title{
Dyson's equations for the (Ising) spin-glass
}

\author{
C. De Dominicis $\left({ }^{1,2}\right)$, I. Kondor $\left({ }^{3}\right)$ and T. Temesvari $\left({ }^{3}\right)$
}

( $\left.{ }^{1}\right)$ CEA, Service de Physique Théorique, CE-Saclay, 91191 Gif-sur-Yvette Cedex, France

(2) Ecole Nationale Supérieure des Mines de Parıs, 75252 Paris, France

$\left({ }^{3}\right)$ Institute for Theoretical Physics, Eötvös University, Budapest, Hungary

(Received 28 March 1994, accepted in final form 7 June 1994)

\begin{abstract}
Résumé . - Un problème complexe est résolu ici : nous établissons pour le verre de spın d'Ising, les équations de Dyson qu relient le propagateur $G$ à l'opérateur de masse $M$. En d'autres termes nous réduisons l'inversion d'une matrice ultramétrique $M$ à la solution d'équations de Dyson, et ceci dans tous les secteurs (ce résultat avait déjà été établi dans le secteur du replicon). Ce qui rend ce problème abordable, c'est l'introduction en lieu et place des composantes de $G$ (ou $M$ ), d'un objet appelé le "noyau" d'où les composantes pourront se déduire après habillage ultramétrique et somme sur les indices de valeurs propres pondérées par leur multiplicité. Les équations de Dyson sont alors établies comme équations de stationnarité de $\operatorname{tr} \ln M-\operatorname{tr} G M$, où les termes cinétiques sont incorporés dans $M$. A chaque étape, le calcul est illustré par le résultat explicite pour le système nu (champ moyen pour $M$ ). En particulier l'introduction du "noyau" permet de construire les propagateurs nus pour un lagrangien comportant tous les invariants quartiques. Enfin le système en présence d'un champ magnétique est aussi résolu.
\end{abstract}

\begin{abstract}
A complex problem is solved here; we show how to write Dyson's equations for the (Ising) spin-glass that relate the propagator $G$ to the mass operator $M$. In other words we are able to reduce the inversion of an ultrametric matrix $M$ to the solution of a Dyson's equation in all sectors (for the replicon sector the result had already been derived). It turns out that what renders the problem tractable is using, instead of the components of $G$ (or $M$ ), an object called here the "kernel" from which one can deduce the components themselves after dressing it with ultrametric weights and summing over eigenvalue indices with their appropriate multiplicity. Dyson's equations are then established as stationarity equations of $\operatorname{tr} \ln M-\operatorname{tr} G M$, where the kinetic terms are incorporated in $M$. At each stage we illustrate the calculation by providing explicit answers for the bare system (mean field in $M$ ). In particular the introduction of the "kernel" allows us to construct the bare propagator for a Lagrangean where one retains all quartic invariants. The case of the system in a magnetic field is also treated.
\end{abstract}

Understanding the properties of the spin glass in the condensed phase has been outstanding as a major challenge for many years. Field theory from the paramagnetic phase has been thoroughly investigated yielding exponents at the critical temperature and above (Harris et 
al. [1], Elderfield and Mc Kane [2], Green [3]). Below and near $T_{\mathrm{c}}\left(\tau=\left(T-T_{\mathrm{c}}\right) / T_{\mathrm{c}} \ll 1\right)$ analytical progress has been much slower. Eigenvalues (De Dominicis and Kondor [4]) of the Hessian around the Parisi solution [5], then multiplicities and bare propagators (De Dominicis and Kondor [6, 7] hereafter referred as I, Kondor and De Dominicis [8] hereafter referred as II, Sompolinsky and Zippelius [9], Goltsev [10]) have been successively obtained. Yet the loop expansion has remained a remote target. The first steps in that direction were made under simplifying circumstances (De Dominicis, Kondor and Temesvari [11]):

(i) the equation of state (i.e. with zero external momentum) was obtained to one loop and even two loops when restricting the internal loop momenta to the order of the large (longitudinal) mass. This permitted a check on scaling and exponentiation below $T_{\mathrm{c}}$.

(ii) the mass operator for the replicon sector was estimated to one loop in the special limit of zero overlap (which is the case with the worst infrared singularity.)

The replicon sector for the propagator is a privileged one since the relationship between propagator and its inverse (which we call mass operator, thus incorporating in it the kinetic terms) is explicitly known. This relationship may be duly called a Dyson's equation. In the other sector, the longitudinal-anomalous one, no such relationship exists so far and it is one of the purposes of this paper to show how it can be established.

To give a feeling of how remote the standard loop expansion was - and still, to some extent, remains, despite the results presented in this work - let us first look at the bare propagator ${ }^{0} G^{\alpha \beta ; \gamma \delta}(p)$. It is calculated as a Gaussian average with the quadratic Lagrangean obtained from the Parisi [5] one

$$
\mathcal{L}_{p}=-\frac{1}{2} \sum_{p} \sum_{(\alpha \beta)} \varphi_{\alpha \beta}(p)\left[p^{2}-2 \tau\right] \varphi_{\alpha \beta}(-p)+\sum_{j}\left[\frac{w}{6} \operatorname{tr} \varphi^{3}(j)+\frac{u}{6} \sum_{(\alpha \beta)} \varphi_{\alpha \beta}^{4}(j)\right]
$$

after the shift

$$
\varphi_{\alpha \beta}(j)=q_{\alpha \beta}+\delta \varphi_{\alpha \beta}(j)
$$

that is, with

$$
\mathcal{L}_{p}^{(2)}=-\frac{1}{2} \sum_{p} \sum_{(\alpha \beta)} \sum_{(\gamma \delta)} \delta \varphi_{\alpha \beta}(p){ }^{0} M^{\alpha \beta ; \gamma \delta}(p) \delta \varphi_{\gamma \delta}(-p) .
$$

Here we use both $\varphi_{\alpha \beta}(j)$ at site $j$ and its Fourier transform $\varphi_{\alpha \beta}(p), \alpha$ and $\beta$ are replicas. The order parameter $q_{\alpha \beta}$ is the Parisi one $q_{\alpha \beta} \longrightarrow q(x)$ for the overlap $(\alpha \cap \beta)=x$. Then we have the bare propagator ${ }^{0} G$, inverse of the bare mass operator ${ }^{0} M$, with

$$
{ }^{0} G^{\alpha \beta ; \gamma \delta}=\left\langle\delta \varphi_{\alpha \beta}(p) \delta \varphi_{\gamma \delta}(-p)\right\rangle_{0} .
$$

and \langle\rangle$_{0}$ standing for the Gaussian integration over $\delta \varphi$. A convenient parametrization turns out to be

$$
{ }^{0} G^{\alpha \beta ; \gamma \delta}={ }^{0} G_{z_{1} ; z_{2}}^{x ; y}
$$

with

$$
\begin{array}{rlr}
(\alpha \cap \beta) & =x \quad(\gamma \cap \delta)=y \\
z_{1} & =\max \{\alpha \cap \gamma ; \alpha \cap \delta\} \\
z_{2} & =\max \{\beta \cap \gamma ; \beta \cap \delta\}
\end{array}
$$

and ultrametric geometry guarantees that of the four overlaps $x, y, z_{1}, z_{2}$ only three are distincts. Suppose we draw the propagator ${ }^{0} G^{\alpha \beta, \gamma \delta}$ as a line

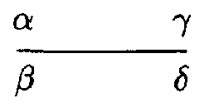


and associate with it the component as in (5).

The formal series giving the full propagator $G^{\alpha \beta ; \gamma \delta}$ in terms of the mass operator can be written out but remains unfortunately out of reach of a direct evaluation. Even the first term of that series, say the term in $w^{2}$ ( $w$ being the marginal cubic coupling) as below

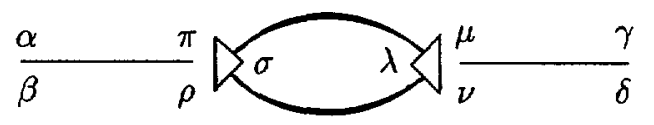

requires excessive algebraic efforts to evaluate it. And in any case what we need is the resummed series to be able to carry out perturbation (or loop) expansion for the mass operator rather than the propagator.

This is enough of a motivation to try to obtain a Dyson's relationship in all sectors (the obvious relationship involving explicit replicas is useless, the only practicable one, so far, being that obtained after letting the number of replicas $n$ go to zero). In the last section we shall return to evaluate the degree of algebraic complexity left to write out the loop expansion, when using the results below.

The terminology we use follows from the work of de Almeida and Thouless [12] who first performed (in the paramagnetic phase) the analysis in eigenvalues and eigenvectors of the quadratic form (3) (see also Refs. [13, 14]). They uncovered what was later called a Longitudinal Anomalous sector, associated with massive modes, and, a Replicon sector, with modes turning massless along the so-called de Almeida-Thouless line. In the following we use the lower left capital index $A$ or $R$ to distinguish the two sectors. That is instead of (5) we now write ${ }_{\mathrm{A}}^{0} G_{z_{1} ; z_{2}}^{x ; y}$ or ${ }_{\mathrm{R}}^{0} G_{z_{1} ; z_{2}}^{x ; x}$.

The starting point of this paper is the propagator structure as given in [I] where the components ${ }_{\mathrm{A}} G_{z_{1} ; z_{2}}^{x ; y}$ are written in terms of a "kernel" $F_{k}^{x ; y}$, and likewise for the replicon components ${ }_{\mathrm{R}} G_{z_{1} ; z_{2}}^{x ; x}$ and associated kernel $F_{k ; \ell}^{x ; x}$. The same "kernel" structure can be written out for the mass operator components ${ }_{\mathrm{A}} M_{z_{1} ; z_{2}}^{x ; y}$ with a kernel $K_{k}^{x ; y}$ (and ${ }_{\mathrm{R}} M_{z_{1} ; z_{2}}^{x ; x}$ with a kernel $K_{k ; \ell}^{x ; x}$ ) where the meaning of the undices $k$ (and $k, \ell$ ) is then related to the eigenvalues of the mass operator matrix.

Admittedly one would like to get some understanding of what the "kernels" are $\left({ }^{1}\right)$. In [I, II] they were written out as the objects that would allow, at the price of some ultrametric dressing, a simple representation of the propagator components. And in the appendix we indeed display the components as a sum over eigenvalue indices, weighted by the corresponding multiplicities, under the constraints of ultrametric geometry, relationships that look quite natural. For some of the components, easily attainable by functional derivation, one can also identify clearly the origin of the kernel structure and this will be done at a couple of instances in the text.

However a general derivation of the component/kernel relationships would require

(i) a complete eigenvector (and multiplicity) analysis which is briefly summarized in [II]

(ii) a block diagonalization of the general ultrametric matrix, through the transform matrix built with the eigenvectors as columns.

The above procedure reduces the original $\frac{n(n-1)}{2} \times \frac{n(n-1)}{2}$ matrix $(G$ or $M)$ into a string of $n \quad(R+1) \times(R+1)$ block diagonal matrices $\left({ }^{2}\right)$ on which one can directly read the kernel $\left(F\right.$ or $K$ ) for the longitudinal-anomalous sector $\left(^{3}\right)$

(1) See also below the remark, concerning the meaning of the kernels, due to Parıs.

$\left({ }^{2}\right)$ The block diagonal $(R+1) \times(R+1)$ matrix $M$ is displayed, in the continuum limit, in section 2 , equation (2.16).

$\left({ }^{3}\right)$ For the replicon sector, the procedure yields $n(n-1) / 2-n(R+1)$ diagonal values directly identified with the kernel. 
This procedure has to be carried out in the discrete and is the subject of a separate paper [15].

The approach adopted here is more practical. The fact that we assume from the start the components vs kernels relationships (as of [I]) allows us to work directly in the continuum. There the eigenvector structure simplifies considerably, thus opening a shortcut to establishing Dyson's equations.

Sections 1,2 are devoted to writing out, in compact form, components vs. kernels and the reverse relationships, kernels vs. components, for the propagators and mass operators.

The introduction of kernels leads to drastically simplified eigenvalue equations and associated determinant of eigenvalues. One can then easily write out a functional $-n \mathcal{F} \equiv \operatorname{tr} \ln \overline{\bar{M}}-\operatorname{tr} \overline{\bar{G}} \overline{\bar{M}}$ in terms of the corresponding kernels (Sect. 3), which under variations of $\overline{\bar{K}}$ (the kernel of $\overline{\bar{M}}$ ) yields the required Dyson's equations (Sect. 4).

Section 5 is dedicated to the soluble bare case where it is shown how the propagator kernel $F$ can explicitly be constructed, even when one keeps, in the Lagrangean, quartic terms like $\operatorname{tr} \varphi^{4}$.

Finally, section 6 is devoted to systems in a magnetic field where it is shown that the relationship giving components ${ }_{\mathrm{A}} G^{x ; y}$ in terms of the kernel $F_{k}^{x ; y}$ contains an extra (surface) term $\delta F_{k=0}^{x ; y}$ which is derived, as above, via a stationarity condition on $\mathcal{F}$. Up to that section, the formalism is kept to a reasonable level of complexity by working directly in the continuum limit. In the last section one has to extract a discontinuity proportional to $n$ as $k$ is going to zero, so that one has to keep track of and work with discrete overlaps.

The continuum limit is what physics requires for the spin glass (i.e. the number of steps of Parisi replica symmetry breaking $R \longrightarrow \infty$ ), to remove all negative masses. But before we start dealing with it, we think it appropriate to open a parenthesis and recall some features of the discrete representation.

We have the successive Parisi boxes of sizes

$$
p_{0} p_{1} . . p_{j} \ldots p_{R} p_{R+1} \quad \text { with } \quad p_{0} \equiv n, \quad p_{R+1} \equiv 1
$$

Then one parametrizes a replica $\alpha$ by the sequence

$$
\begin{gathered}
\alpha:\left\{\theta_{1}, \theta_{2}, ., \theta_{R+1}\right\} \quad \text { with } \\
\theta_{1}=1,2, ., p_{0} / p_{1} ; \quad \theta_{2}=1,2, . ., p_{1} / p_{2} ; \cdot ; \theta_{R+1}=1,2, \ldots, p_{R} / p_{R+1} .
\end{gathered}
$$

Two replicas $\alpha, \beta$ given by $\left\{\theta^{\alpha}\right\},\left\{\theta^{\beta}\right\}$ have an overlap $(\alpha \cap \beta)=r$ if $\theta_{j}^{\alpha}=\theta_{j}^{\beta}$ for $j \leq r$ and $\theta_{r+1}^{\alpha} \neq \theta_{r+1}^{\beta}$.

In the continuum limit $\frac{r}{R+1} \longrightarrow x, 0 \leq x \leq 1$, and by extension $x$ is also called the overlap. Likewise $p_{r} \longrightarrow p(x)$ and in the Parısi gauge one chooses $p(x)=x$, hence $p_{r+1}-p_{r} \longrightarrow \mathrm{d} x$.

Thus for example the sum over pairs $(\alpha \beta)$ of $q_{\alpha \beta}^{2}$ becomes

$$
\sum_{\alpha<\beta} q_{\alpha \beta}^{2}=\frac{1}{2} \sum_{\alpha, \beta} q_{\alpha \beta}^{2} \longrightarrow \frac{n}{2} \sum_{r=0}^{R}\left(p_{r}-p_{r+1}\right) q_{r}^{2} \longrightarrow-\frac{n}{2} \int_{0}^{1} \mathrm{~d} x q^{2}(x)
$$

where the first step is for the discrete Parisi representation and the second for the continuum limit in the Parisi gauge. Unless explicitly stated these two steps are always implied in the following.

Turnıng to eigenvalues and eigenvectors, in the longitudinal anomalous sector, eigenvalues are indexed by $k=0$ (longitudinal) $k=1,2,, R+1$ (anomalous). The eigenvectors are taken 
as $f_{\alpha \beta}^{\mu} \longrightarrow f_{z}(x)$ with $(\alpha \cap \beta)=x$ and $z=\max \{(\mu \cap \alpha) ;(\mu \cap \beta)\}$, where the only non vanishing component in this continuum limit is $f_{k}(x)$. The corresponding multiplicity $\mu(k)$ is

$$
\frac{1}{n} \mu(k)=\frac{1}{p_{k}}-\frac{1}{p_{k-1}} \longrightarrow-\frac{\mathrm{d} k}{k^{2}}
$$

where for the sole eigenvalue indices ( $k$ here; $k, \ell$ below) we keep, for lack of better, the same symbol for discrete and continuous variables.

In the replicon sector, eigenvalues are indexed by $k, \ell$ and the eigenvectors $f_{\alpha \beta}^{\mu \nu}$ with $(\mu \cap \nu)=$ $r<k, \ell$ are not needed in the following. The multiplicities, in discrete form, are now [II].

$$
\begin{aligned}
& \frac{p_{r}}{2 p_{r+1}}\left(\frac{1}{p_{r+1}}-\frac{1}{p_{r}}\right)-\sum_{k=0}^{r+1} \frac{1}{n} \mu(k) \quad \text { if } \quad k=\ell=r+1 \\
& \frac{1}{n} \mu(r ; k, \ell)=\frac{1}{2 p_{r+1}}\left(p_{r}-p_{r+1}\right)\left(\frac{1}{p_{k}}-\frac{1}{p_{k-1}}\right)-\frac{1}{2 n} \mu(k) \quad \begin{array}{lll}
k>r+1 \\
\text { if } & \begin{array}{l}
\ell=r+1 \\
k \longleftrightarrow \ell
\end{array}
\end{array} \\
& \frac{1}{2}\left(p_{r}-p_{r+1}\right)\left(\frac{1}{p_{k}}-\frac{1}{p_{k-1}}\right)\left(\frac{1}{p_{\ell}}-\frac{1}{p_{\ell-1}}\right) \quad \text { if } \quad k, \ell<r+1
\end{aligned}
$$

The terms containing $\mu(k)$ (the "singular" component) will be ignored until section 3 . For the rest, the "regular" component of the multiplicity, we can now write the continuum limit as

$$
\frac{1}{n} \mu_{\text {reg }}(x ; k, \ell)=-\frac{1}{2} \mathrm{~d} x \frac{\mathrm{d} k}{k^{2}} \frac{\mathrm{d} \ell}{\ell^{2}}(\Theta(k-x)-k \delta(k-x)) \cdot(\Theta(\ell-x)-\ell \delta(\ell-x))
$$

Note that in the following, when integrating $\mu(k)$ or $\mu(x ; k, \ell)$ we shall, for convenience, keep using the summation symbol $\sum_{k}$ or $\sum_{x, k, \ell}$. With this minimal package, we now proceed.

\section{The replicon sector.}

In this sector the eigenvalue equations reduce to algebraic equations [4]. As a consequence writing the Dyson equation relating propagator components and mass operator is a relatively simple enterprise.

1.1 Propagators. - The propagator expressions in terms of their kernels are taken from [I]. In order to retain some intuitive meaning for the compact equations written below, we exhibit them, in the appendix, as summations weighted by multiplicities and ultrametric factors. In the same appendix we also write them in fully explicit form.

The expressions introduced below have not only the advantage of compactness, they turn out to involve the precise operator expressions that appear when use is made of the $p$-adic representation $\left({ }^{4}\right)$. form

Calling $F_{k ; \ell}^{x ; x}$ the kernel associated with the replicon propagator we can write in compact

$$
{ }_{\mathrm{R}} G_{z_{1} ; z_{2}}^{x ; x}=\int_{x}^{z_{1}} D_{k} \int_{x}^{z_{2}} D_{\ell} F_{k ; \ell}^{x ; x}
$$

$\left({ }^{4}\right)$ According to Parisi, private communication, the kernels are the $p$-adic Fourier transform of the components as read off in equations $(1.1,2)$ below. In the longitudinal-anomalous sector the relationship is not yet understood. 
with

$$
D_{k}=\left(-\frac{1}{k} \frac{\partial}{\partial k}+\delta\left(k-1_{-}\right)\right) \mathrm{d} k
$$

The $\delta$ function contributes only when the $z$ upper bound is exactly one (i.e. for a single pair of coinciding replicas as in ${ }_{\mathrm{R}} G^{\alpha \beta ; \alpha \gamma} \equiv{ }_{\mathrm{R}} G_{1 ; z}^{x ; x}$ or for a double coincidence as in ${ }_{\mathrm{R}} G^{\alpha \beta ; \alpha \beta} \equiv$ $\left.{ }_{R} G_{1 ; 1}^{x ; x}\right)$. Note that above the breakpoint $x_{1}\left(q\left(k \geq x_{1}\right) \equiv q_{1}\right)$ there is no more dependence upon $k$, hence the integrand associated with $\partial / \partial k, k>x_{1}$, vanishes (likewise for $\ell$ ). Eqs. $(1.1,2$ ) are contained in [6] and [I]) and for the $x=0$ limit in (Sompolinsky and Zippelius [9], Goltsev [10]).

The inverse relationship may be written as

$$
F_{k ; \ell}^{x ; x}=\int_{k}^{1+} \mathcal{D}_{z_{1}} \int_{\ell}^{1+} \mathcal{D}_{z_{2}} G_{z_{1} ; z_{2}}^{x ; x}
$$

with

$$
\mathcal{D}_{z}=\left(-z \frac{\partial}{\partial z}+\delta\left(z-1_{-}\right)-\delta(z-1)\right) \mathrm{d} z
$$

Again we have chosen to write it in a compact form. Equation (1.3) can be directly checked by subtitution into (1.1). The $\delta$ functions bring in the discontinuity of the propagators between $1_{-}$(or $x_{1}$, since above $x_{1}$ there is no $z$ dependence) and 1 i.e. $G_{1 ; x_{1}}^{x ; x}-G_{1 ; 1}^{x ; x}$ or $G_{x_{1} ; x_{1}}^{x ; x}-G_{1 ; x_{1}}^{x ; x}$. For $z>x_{1}$ the terms in $z \partial / \partial z$ do not contribute. Note that on the right hand side of (1.3) we have omitted the left subscript $R$. We shall see below why this is justified.

1.2 MASS OPERATORS. - The above relationship between components and kernels apply also to the mass operator ${ }_{\mathrm{R}} M_{z_{1} ; z_{2}}^{x ; x}$ and its kernel $K_{k ; \ell}^{x ; x}$ giving instead of $(1.1,1.3)$ :

$$
\begin{gathered}
{ }_{\mathrm{R}} M_{z_{1} ; z_{2}}^{x ; x}=\int_{x}^{z_{1}} D_{k} \int_{x}^{z_{2}} D_{\ell} K_{k ; \ell}^{x ; x} \\
K_{k ; \ell}^{x ; x}=\int_{k}^{1+0} \mathcal{D}_{z_{1}} \int_{\ell}^{1+0} \mathcal{D}_{z_{2}} M_{z_{1} ; z_{2}}^{x ; x}
\end{gathered}
$$

and $D_{k}$ as in (1.2), $\mathcal{D}_{z}$ as in (1.4). The right hand side of (1.6) can be directly read off the replicon eigenvalue equation, identifying $K_{k ; \ell}^{x ; x}$ as the replicon eigenvalue with multiplicity $\mu(x ; k, \ell)$; we shall also use the notation displaying the kinetic term,

$$
K_{k ; \ell}^{x ; x} \equiv p^{2}+\lambda_{p}(x ; k, \ell)
$$

Consider now the equation of state for an overlap $(\alpha \cap \beta)=x$

$$
0=\left.\frac{\partial}{\partial q_{\alpha \beta}} f\left\{q_{\alpha \beta}\right\}\right|_{(\alpha \cap \beta)=x}=\frac{\delta f}{\delta q(x)}
$$

This is true for all $x$ and hence for the derivative $\left(x<x_{1}\right)$

$$
0=\frac{1}{\dot{q}} \frac{\mathrm{d}}{\mathrm{d} x} \frac{\delta f}{\delta q(x)}
$$


which can be written

$$
\begin{aligned}
0 & =\left\{\frac{\partial^{2} f}{\partial q_{\alpha \beta} \partial q_{\alpha \beta}}+\left.\sum_{\gamma} \frac{\partial^{2} f}{\partial q_{\alpha \gamma} \partial q_{\alpha \beta}}\right|_{(\alpha \cap \gamma)=x}+\left.\sum_{\delta} \frac{\partial^{2 f}}{\partial q_{\beta \delta} \partial q_{\alpha \beta}}\right|_{(\beta \cap \delta)=x}\right. \\
& \left.+\left.\sum_{(\gamma \delta)} \frac{\partial^{2} f}{\partial q_{\gamma \delta} \partial q_{\alpha \beta}}\right|_{(\gamma \cap \delta)=x}\right\}_{(\alpha \cap \beta)=x}
\end{aligned}
$$

Since

$$
M^{\alpha \beta ; \gamma \delta}(p=0) \equiv \frac{\partial^{2}(-\beta f)}{\partial q_{\alpha \beta} \partial q_{\gamma \delta}}
$$

one immediately recovers a special case of (1.6), viz

$$
\begin{gathered}
0=K_{x ; x}^{x ; x}(0)=M_{1 ; 1}^{x ; x}(0)-2\left[x M_{1 ; x}^{x ; x}(0)+\int_{x}^{1} \mathrm{~d} z M_{1 ; z}^{x ; x}(0)\right]+x^{2} M_{x ; x}^{x ; x}(0)+2 x \int_{x}^{1} \mathrm{~d} z M_{x ; z}^{x ; x}(0) \\
+\int_{x}^{1} \mathrm{~d} z_{1} \int_{x}^{1} \mathrm{~d} z_{2} M_{z_{1} ; z_{2}}^{x ; x}(0)
\end{gathered}
$$

thereby exhibiting a zero mode, for all $x$,

$$
K_{x ; x}^{x ; x}(0) \equiv \lambda_{p=0}(x ; x, x)=0 .
$$

Note that if, instead of summing over $\gamma, \delta$ solely restricted to the constraints imposed in (1.10), we choose $k, \ell>x$ and impose $\max \{(\alpha \cap \gamma) ;(\alpha \cap \delta)\} \equiv z_{1} \geq k$ and $\max \{(\beta \cap \gamma) ;(\beta \cap \delta)\} \equiv z_{1} \geq \ell$ we explicitly construct the eigenvalue (at zero momentum):

$$
K_{k ; \ell}^{x ; x}(0) \equiv \lambda_{p=0}(x ; k, \ell)=\int_{k}^{1} \mathcal{D} z_{1} \int_{\ell}^{1} \mathcal{D} z_{2} M_{z_{1} ; z_{2}}^{x ; x}(p=0)
$$

To end this section with a simple illustration, let us look at the bare mass operator matrix, i.e. the Hessian associated with the Parisi Lagrangean after the shift $\varphi_{\alpha \beta}(j)=q_{\alpha \beta}+\delta \varphi_{\alpha \beta}(j)$ as of equation (3). One finds immediately

$$
\begin{aligned}
& { }^{0} M^{\alpha \beta ; \alpha \beta}(p)=p^{2}-2 \tau-2 u q_{\alpha \beta}^{2} \\
& { }^{0} M^{\alpha \beta ; \alpha \gamma}(p)=-w q_{\beta \gamma} \\
& { }^{0} M^{\alpha \beta ; \gamma \delta}(p)=0
\end{aligned}
$$

Then, from (1.6) or (1.4) one gets

$$
{ }^{0} K_{k ; \ell}^{x ; x}(p)=p^{2}+\lambda_{p}^{0}(x ; k, \ell)=p^{2}-2\left(\tau+u q^{2}(x)\right)+E^{0}(k)+E^{0}(\ell)
$$

where

$$
\begin{aligned}
& E^{0}(k)=w\left[k q(k)+\int_{k}^{1} \mathrm{~d} s q(s)\right] \quad k>x \\
& E^{0}(k)=E^{0}(x) \quad k \leq x
\end{aligned}
$$

Note that from the equation of state

$$
0=-2\left[\tau+u q^{2}(x)-E^{0}(x)\right]
$$


Having the replicon eigenvalues we can write their contribution to the free energy (at zero loop order) as

$$
-\beta_{\mathrm{R}} f_{0} n=-\frac{1}{2} \sum_{p} \sum_{x, k, \ell} \mu_{\mathrm{reg}}(x ; k, \ell) \ln \left[p^{2}+\lambda^{0}(x ; k, \ell)\right]
$$

with $\mu_{\text {reg }}(x ; k, \ell)$ as in (12). If one remembers that (1.19) is also generated as the Gaussian integral over $\delta \varphi$ on the quadratic from $-\frac{1}{2} \operatorname{tr} \delta \varphi^{0} \overline{\bar{M}}(p) \delta \varphi$, then by taking a derivative over ${ }^{0} M^{\alpha \beta ; \alpha \beta}(p)$, one obtains

$$
\left.{ }_{\mathrm{R}}^{0} G^{\alpha \beta ; \alpha \beta}(p)\right|_{(\alpha \cap \beta)=x} \equiv-\frac{n}{2} \mathrm{~d} x{ }_{\mathrm{R}}^{0} G_{1 ; 1}^{x ; x}(p)=\sum_{k, \ell} \mu_{\mathrm{reg}}(x ; k, \ell) \frac{1}{p^{2}+\lambda^{0}(x ; k, \ell)}
$$

which coincides with $(1.1,2)$ after partial integrations over $k, \ell$. Hence at a small price, we are displaying the Dyson relationship for the (bare) replicon

$$
F_{k ; \ell}^{x ; x}=1 / K_{k ; \ell}^{x ; x}
$$

where we have omitted the zero (bare) superscript, since the same derivation applies to the full replicon propagator and mass operator. We shall return to the derivation of (1.21) in sections 3,4 .

\section{The longitudinal anomalous sector.}

This is the sector where we aim to derive a Dyson equation. We give here the relationships between the propagator $G$ (or mass operator $M$ ) and its kernel $F$ (or $K$ ). See also the appendix and section 3 .

\subsection{PROPAGators.}

$$
\begin{aligned}
& x \leq y \leq t \quad G_{x ; t}^{x ; y}=\left(\int_{0}^{x}+\frac{1}{2} \int_{x}^{y}+\frac{1}{4} \int_{y}^{t}\right) D_{k} F_{k}^{x ; y} \\
& x \leq t \leq y \quad G_{x ; t}^{x ; y}=G_{t ; t}^{x ; y}=\left(\int_{0}^{x}+\frac{1}{2} \int_{x}^{t}\right) D_{k} F_{k}^{x ; y} \\
& t \leq x \leq y \quad G_{t ; t}^{x ; y}=\int_{0}^{t} D_{k} F_{k}^{x ; y}
\end{aligned}
$$

and $D_{k}$ as in (1.2). The components are not restricted to be diagonal as in the replicon sector (hence we may omit the subscript $A$ from the left hand side). The only missing component is ${ }_{\mathrm{A}} G_{z_{1} ; z_{2}}^{x ; x}$ which we can write in terms of $(2.1)$ itself

$$
x \leq z_{1}, z_{2} \quad{ }_{\mathrm{A}} G_{z_{1} ; z_{2}}^{x ; x}=G_{x ; z_{2}}^{x ; x}+G_{z_{1} ; x}^{x ; x}-G_{x ; x}^{x ; x}
$$

with $G_{x ; z}^{x ; x}$ obtained from (2.1a). The subscript A is not needed on the right hand side since ${ }_{\mathrm{R}} G_{z_{1} ; z_{2}}^{x} ; x$ vanishes for $z=x$.

Again, the $\delta$ function implied in $D_{k}(1.2)$ contributes for a (single) pair of coinciding replicas occurring when $t \equiv 1$ (in (2.1a)), the double coinciding case being contained in (2.2). If $k>x_{1}$, 
the integrand associated with the $\partial / \partial k$ piece of $D_{k}$ vanishes. Finally, it should be noted that equations (2.1) are to be modified (with a surface term added) in the presence of a magnetic field (Sect. 6).

The inverse relationships are

$$
\begin{aligned}
& k<x \leq y \quad-\frac{1}{4} F_{k}^{x ; y}=\frac{1}{4} \int_{k}^{x} \mathcal{D}_{t} G_{t ; t}^{x ; y}+\left(\frac{1}{2} \int_{x}^{y}+\int_{y}^{1+}\right) \mathcal{D}_{t} G_{x ; t}^{x ; y} \\
& x<k<y \quad-\frac{1}{4} F_{k}^{x ; y}=\left(\frac{1}{2} \int_{k}^{y}+\int_{y}^{1+}\right) \mathcal{D}_{t} G_{x ; t}^{x ; y} \\
& x \leq y<k \quad-\frac{1}{4} F_{k}^{x ; y}=\int_{k}^{1+} \mathcal{D}_{t} G_{x ; t}^{x ; y}
\end{aligned}
$$

and can be checked by substitution into (2.1). $\mathcal{D}_{t}$ is as in (1.4), and the $\delta$ functions contribute equally to $a, b, c$; so that (2.3) could also be written with (i) $-t \mathrm{~d} t \frac{\partial}{\partial t}$ instead of $\mathcal{D}_{t}$, (ii) $\int^{1-}$ instead of $\int^{1+}$, (iii) adding $G_{x ; x_{1}}^{x ; y}-G_{x_{;} ;}^{x ; y}$ to each equation.

One remarkable feature is that, if one uses $(2.1,2)$ to build ${ }_{A} G_{z_{1} ; z_{2}}^{x ; x}$ and insert it into the right hand side of (1.3), it results in a null contribution to $F_{k ; \ell}^{x ; x}$

$$
0=\int_{k}^{1+} \mathcal{D}_{z_{1}} \int_{\ell}^{1+} \mathcal{D}_{z_{2} A} G_{z_{1} ; z_{2}}^{x ; x}
$$

I e. $\int_{k}^{1+} \mathcal{D}_{z_{1}} \int_{k}^{1+} \mathcal{D}_{z_{2}}$ acts as a projector onto the replicon subspace. This is why the subscript $\mathrm{R}$ can be omitted in (1.3).

2.2 MASS OPERATORS. - Just like the above, in the replicon subspace, the structural relationship relating the pair $G, F$ remained valid for the pair $M, K$, so likewise we can rewrite (2.1-4) for the mass operator pair. For reference we just quote the last equations (c):

$$
t \leq x \leq y \quad M_{t ; t}^{x ; y}=\int_{0}^{x} D_{k} K_{k}^{x ; y} \quad(c) \text { etc... }
$$

and

$$
\begin{gathered}
x \leq y<k \quad-\frac{1}{4} K_{k}^{x ; y}=\int_{k}^{1+} \mathcal{D}_{t} M_{x ; t}^{x ; y} \quad(c) \quad \text { etc... } \\
{ }_{\mathrm{A}} M_{z_{1} ; z_{2}}^{x ; x}=M_{x ; z_{2}}^{x ; x}+M_{z_{1} ; x}^{x ; x}-M_{x ; x}^{x ; x}
\end{gathered}
$$

Before proceeding, let us illustrate the above by giving the table of components and kernels for the bare mass operator.

$$
\begin{aligned}
& { }^{0} M_{1 ;}^{x ; y}=-w q(\min x, y) \\
& { }_{A}^{0} M_{1 ; z}^{x ; x}=-w q(x) \\
& { }_{\mathrm{R}}^{0} M_{1 ; z}^{x ; x}=-w(q(z)-q(x)) \\
& { }_{\mathrm{A}}^{0} M_{1 ; 1}^{x ; x}=-2 w q(x) \\
& { }_{\mathrm{R}}^{0} M_{1 ; 1}^{x ; x}=+2 w q(x)+p^{2}-2 \tau-2 u q^{2}(x) \\
& { }^{0} K_{k}^{x ; y}=-4 w q(\min (x, y))
\end{aligned}
$$


and ${ }^{0} K_{k ; \ell}^{x ; x}$ as in (1.16). Note that ${ }^{0} K_{k}^{x ; y}$ is $k$ independent.

Contrary to the replicon sector where we could relate the kernel to the equation of state and variations thereof, in the longitudinal anomalous sector there is no substitute to the hard way i.e. we have to write the eigenvalue equations. So let us start with the bare system:

In the longitudinal case $(k=0)$ we have

$$
\sum_{(\gamma \delta)}{ }^{0} M^{\alpha \beta ; \gamma \delta} f_{\gamma \delta}=\lambda_{0} f_{\alpha \beta}
$$

which with (1.15) writes, for $(\alpha \cap \beta)=x, f_{\alpha \beta} \longrightarrow f_{0}(x)$ :

$$
\begin{aligned}
\left\{p^{2}-2 \tau-2\right. & \left.2 u q^{2}(x)+2 w\left[x q(x)+\int_{x}^{1} \mathrm{~d} t q(t)\right]\right\} f_{0}(x) \\
& +2 w \int_{0}^{x} \mathrm{~d} t q(t) f_{0}(t)+2 w q(x) \int_{x}^{1} \mathrm{~d} t f(t)=\lambda_{0} f(x)
\end{aligned}
$$

Using (1.16) and (2.8), equation (2.10) becomes

$$
{ }^{0} K_{x ; x}^{x ; x} f_{0}(x)-\int_{0}^{1} \mathrm{~d} t{ }^{0} K_{0}^{x ; t} f_{0}(t)=\lambda_{0} f_{0}(x)
$$

In the anomalous case $(k>0)$ we have

$$
\sum_{(\gamma \delta)}{ }^{0} M^{\alpha \beta ; \gamma \delta} f_{\gamma \delta}^{\mu}=\lambda_{k} f_{\alpha \beta}^{\mu}
$$

In the continuum, the only surviving component is such that

$$
f_{\gamma \delta}^{\mu} \longrightarrow f_{k}(t) \quad \text { with }(\gamma \cap \delta)=t, \quad \max \{(\mu \cap \gamma) ;(\mu \cap \delta)\}=k,
$$

With notations of $(2.8,11)$, the eigenvalue equations take the simple form

$$
\begin{aligned}
& k<x \quad{ }^{0} K_{x ; x}^{x ; x} f_{k}(x)-\frac{1}{2} \int_{0}^{1} \mathrm{~d} t{ }^{0} K_{k}^{x ; t} f_{k}(t)-\frac{1}{2}{ }^{0} K_{k}^{x ; k} k f_{k}(k-0)=\lambda_{0} f_{k}(x) \\
& x<k \quad{ }^{0} K_{x ; k}^{x ; x} f_{k}(x)-\frac{1}{4} \int_{0}^{1} \mathrm{~d} t{ }^{0} K_{k}^{x ; t} f_{k}(t)-\frac{1}{4}{ }^{0} K_{k}^{x ; k} k f_{k}(k-0)=\lambda_{0} f_{k}(x)
\end{aligned}
$$

Here it is necessary to specify $f_{k}(k-0)$, because, as can be checked by letting $x$ go to $k$ in $(2.13,14), f_{k}(x)$ has a discontinuity at $x=k$ which gives

$$
f_{k}(k-0)=\frac{1}{2} f_{k}(k+0)
$$

On $(2.13,14)$ one can read off the determinant giving the product of $k$-eigenvalues $\overline{\boldsymbol{\Delta}}_{k}$. It can be simplified by the following trick: multiply all lines up to and including $k-0$ by 2 , and all columns up to and including $k-0$ by $1 / 2$ (i.e. take $f_{k}(t)=\frac{1}{2} \varphi_{k}(t)$ for $t<k$ and $f_{k}(t)=\varphi_{k}(t)$ for $t>k$ ), this operation leaves $\overline{\boldsymbol{\Delta}}_{k}$ invariant. Then keeping in mind that $K_{x ; k}^{x ; x}=K_{x ; x}^{x ; x}$ for $k \leq x$, equations $(2.13,14)$ become one single equation

$$
{ }^{0} K_{x ; k}^{x ; x} \varphi_{k}(x)-\int_{0}^{1} \frac{\Delta_{t}^{(k)}}{2}{ }^{0} K_{k}^{x ; t} \varphi_{k}(t)=\lambda_{k} \varphi_{k}(x)
$$


with the ultrametric weight $\Delta_{t}^{(k)}$ (in its continuous version)

$$
\Delta_{t}^{(k)} \equiv \mathrm{d} t\left[\frac{1}{2} \Theta(k-t)+\frac{k}{2} \delta(k-t)+\Theta(t-k)\right] \equiv \mathrm{d} t U_{k}(t)
$$

Turning to the full mass operator $M^{\alpha \beta ; \gamma \delta}$ one obtains first a very complicated pair of equations [II] (it takes nine lines to spell them out). However, if one expresses $M$ in terms of its kernel $K$, the pair of equations collapses and after use of the above transform $\left(f_{k}(t) \longrightarrow \varphi_{k}(t)\right.$ a function now continuous at $t=k$ ) it reduces to $(2.16,17)$ (with the left superscript zero removed)!

\section{The generating functional.}

We wish now to form the quantity

$$
-n \mathcal{F}=\operatorname{tr} \ln \overline{\bar{M}}-\operatorname{tr} \overline{\bar{G}} \overline{\bar{M}}
$$

which under stationarity with respect to $\overline{\bar{M}}$ generates the generalized Dyson's equation

$$
\overline{\bar{G}}=\bar{M}^{-1}
$$

\subsection{THE LOGARITHMIC TERM.}

$$
\mathcal{L} \equiv \operatorname{tr} \ln \overline{\bar{M}} \equiv \mathcal{L}_{\mathrm{R}}+\mathcal{L}_{\mathrm{LA}}
$$

For $\mathcal{L}_{\mathrm{LA}}$ we need to compute the determinant of $k$-eigenvalues and use $\operatorname{tr} \ln =\ln$ det. So let us write, reading off $(2.16,17)$

$$
\bar{\Delta}_{k} \equiv \prod_{x} K_{x ; k}^{x ; x} \Delta_{k}=\operatorname{det}\left\{K_{x ; k}^{x ; x} \mathbb{1}-K_{k}^{x ; y} \frac{\Delta_{y}^{(k)}}{2}\right\}
$$

Hence

$$
\mathcal{L}_{\mathrm{LA}}=\sum_{x, k} \mu(k) \ln K_{x ; k}^{x ; x}+\sum_{k} \mu(k) \operatorname{tr} \ln \left\{\mathbb{1}-\frac{1}{K_{x ; k}^{x ; x}} K_{k}^{x ; y} \frac{\Delta_{y}^{(k)}}{2}\right\}
$$

with $\mu(k)$ the multiplicity (10) and the ln expansion

$$
\ln \{\}=-\sum_{x} \frac{1}{K_{x ; k}^{x ; x}}\left\{K_{k}^{x ; x}+\frac{1}{2} \sum_{t} K_{k}^{x ; t} \frac{\Delta_{t}^{(k)}}{2 K_{t ; k}^{t ; t}} K_{k}^{t ; x}+\frac{1}{3} \sum_{t, t^{\prime}} . .\right\} \frac{\Delta_{x}^{(k)}}{2}
$$

For $\mathcal{L}_{\mathbf{R}}$ we get

$$
\mathcal{L}_{\mathrm{R}}=\sum_{x, k, \ell} \mu_{\mathrm{reg}}(x ; k, \ell) \ln K_{k ; \ell}^{x ; x}+\sum_{k} \mu_{\text {sing }}(k) \ln K_{x ; k}^{x ; x}
$$

where the contribution in $\mu_{\text {sing }}(k)$ is identically cancelled by the first term in $\mathcal{L}_{\mathrm{LA}}$ (remember $K_{x ; k}^{x ; x}=K_{x ; x}^{x ; x}$ for $\left.k \leq x\right)$. Hence

$$
\mathcal{L} \equiv \operatorname{tr} \ln \overline{\bar{M}}=\sum_{k} \mu(k) \operatorname{tr} \ln \left\{\mathbb{1}-\frac{1}{K_{x ; k}^{x ; x}} K_{k}^{x ; y} \frac{\Delta_{y}^{(k)}}{2}\right\}+\sum_{x, k, \ell} \mu_{\text {reg }}(x ; k, \ell) \ln K_{k ; \ell}^{x ; x} .
$$


3.2 THE $\operatorname{tr} \overline{\bar{G}} \overline{\bar{M}}$ TERM . - A straightforward but lengthy calculation yields tr $\overline{\bar{G}} \overline{\bar{M}}$ in terms of the kernels $F$ and $K$ viz.

$$
\begin{aligned}
-\operatorname{tr} \overline{\bar{G}} \overline{\bar{M}} & =-\sum_{x}\left\{\sum_{k, \ell} \mu_{\mathrm{reg}}(x ; k, \ell) F_{k ; \ell}^{x ; x} K_{k ; \ell}^{x ; x}+\sum_{k} \mu(k) \sum_{t} F_{k}^{x ; t} \frac{\Delta_{t}^{(k)}}{2} K_{k}^{t ; x} \frac{\Delta_{x}^{(k)}}{2}\right. \\
& \left.+\sum_{k} \mu(k)\left[F_{k}^{x ; x} K_{x ; k}^{x ; x}+F_{x ; k}^{x ; x} K_{k}^{x ; x}\right] \frac{\Delta_{x}^{(k)}}{2}\right\}
\end{aligned}
$$

where one recognizes a pure replicon term (the first one) and a pure anomalous term (the second one). Equations $(3.8,9)$ give the generating functional (3.1).

\section{Stationarity equations.}

Notice that we know both the relationships that express the components $G$ (or $M$ ) versus the kernels $F$ (or $K$ ) equations. $(1.1,2) ;(2.1,2)$ (or $(1.5) ;(2.5,7)$ ) and the converse relationships $(1.3,4) ;(2.3)$ (or $(1.6) ;(2.6))$. Thus we could have left $\operatorname{tr} \overline{\bar{G}} \overline{\bar{M}}$ expressed in terms of the components $G, M$ and then understand the $K$ appearing in $\operatorname{tr} \ln \overline{\bar{M}},(3.8)$ as a function of the $M$ components via (1.6), (2.6). As a result we can write stationarity in two different ways:

(i) by differentiating $\mathcal{F}$ with respect to $K$, we obtain $F$ versus $K$

(ii) by differentiating with respect to $M$ (and using the chain rule through $K$ in the ln term) we produce $G$ versus $K$, or, if we use the result of (i), versus $F$ i.e. we have a consistency check.

Another way to view this result is to imagine adding a source term $\overline{\bar{\varepsilon}}$ to the mass operator $\overline{\bar{M}}$. Then stationarity with respect to the $\varepsilon$ components produces the propagator components $G$, generalizing the way we derived above ${ }_{\mathrm{R}} G_{1 ; 1}^{x ; x}$ in $(1.20)$ by differentiating with respect to ${ }^{0} M^{\alpha \beta ; \alpha \beta}(p)$ (simulating $\varepsilon_{1 ; 1}^{x ; x}$ ). Here however we have the kernels of $\varepsilon$ (i.e. $\varepsilon_{k}^{x ; y}, \varepsilon_{x ; k}^{x ; x}$ ) occurring in the log term and hence we have to use the inversion formulae (like (2.6)) and the chain rule to obtain $G$ in terms of its kernel $F$.

\subsection{STATIONARITY VERSUS THE KERNELS $K$.}

- $\delta / \delta K_{k ; \ell}^{x ; x}$

$$
0=\mu_{\mathrm{reg}}(x ; k, \ell)\left[\frac{1}{K_{k ; \ell}^{x ; x}}-F_{k ; \ell}^{x ; x}\right]
$$

which derives the Dyson's equation in the replicon sector (cf. (1.20)).

- $\delta / \delta K_{k}^{y ; x}$

$$
0=\mu(k) \frac{\Delta_{x}^{(k)}}{2}\left[-\frac{1}{K_{x ; k}^{x ; x}}\left\{K_{k}^{x ; y}+\int_{0}^{1} K_{k}^{x ; t} \frac{\Delta_{t}^{(k)}}{2 K_{t ; k}^{t ; t}} K_{k}^{t ; y}+. .\right\} \frac{1}{K_{y ; k}^{y ; y}}-F_{k}^{x ; y}\right] \frac{\Delta_{y}^{(k)}}{2}
$$

For short let us write $K_{x ; k}^{x ; x} \equiv \Lambda_{k}(x)$, then the above equation becomes

$$
-\int_{0}^{1} K_{k}^{x ; t} \frac{\Delta_{t}^{(k)}}{2} F_{k}^{t ; y}+\Lambda_{k}(x) F_{k}^{x ; y}+K_{k}^{x ; y} \frac{1}{\Lambda_{k}(y)}=0
$$


This is the Dyson equation in the anomalous sector (solved by iteration in (4.2)) that relates (the kernel of) the propagator $F$ to (the kernel of) the mass operator $K$.

It is convenient to write it as the following integral equation

$$
\hat{F}_{k}^{x ; y}=K_{k}^{x ; y}+\int_{0}^{1} K_{k}^{x ; t} \frac{\Delta_{t}^{(k)}}{2 \Lambda_{k}(t)} \hat{F}_{k}^{t ; y}
$$

where $\hat{F}$ is related to $F$ via

$$
\hat{F}_{k}^{x ; y}=-\Lambda_{k}(x) F_{k}^{x ; y} \Lambda_{k}(y)
$$

- $\delta / \delta K_{x ; k}^{x ; x}$ : This stationarity condition yields (4.2) in the $y \longrightarrow x$ limit showing that $F_{k}^{x ; x}=$ $\lim _{y \longrightarrow x} F_{k}^{x ; y}$ Finally $\delta / \delta K_{k}^{x ; x}$ does not produce anything new.

4.2 STATIONARITY VERSUS COMPONENTS $M$. - In order to play this part of the game we have to rewrite $\operatorname{tr} \overline{\bar{G}} \overline{\bar{M}}$ in terms of components (rather than kernels as in (3.9)); we get

$$
\begin{aligned}
-\operatorname{tr} \overline{\bar{G}} \tilde{\bar{M}} & =n\left\{\int_{0}^{1} \mathrm{~d} x \int_{x}^{1} \mathcal{D}_{s} \int_{x}^{1} \mathcal{D}_{t} G_{s ; t}^{x ; x} M_{t ; s}^{x ; x}\right. \\
& +\frac{1}{2} \int_{0}^{1} \mathrm{~d} x \int_{0}^{1} \mathrm{~d} y\left(\int_{x}^{1} \mathcal{D}_{s} G_{x ; s}^{x ; y} M_{s ; x}^{y ; x}+\int_{y}^{1} \mathcal{D}_{s} G_{y ; s}^{y ; x} M_{s ; y}^{x ; y}\right) \\
& +\frac{1}{8} \int_{0}^{1} \mathrm{~d} x \int_{0}^{1} \mathrm{~d} y\left(\int_{0}^{x} \mathcal{D}_{s} G_{s ; s}^{x ; y} M_{s ; s}^{y ; x}+\int_{0}^{y} \mathcal{D}_{s} G_{s ; s}^{y ; x} M_{s ; s}^{x ; y}\right) \\
& \left.+\frac{1}{4} \int_{0}^{1} \mathrm{~d} x \int_{0}^{x} \mathrm{~d} y \int_{y}^{x} \mathcal{D}_{s} G_{s ; s}^{x ; y} M_{s ; s}^{y ; x}+\frac{1}{2} \int_{0}^{1} \mathrm{~d} x \int_{x}^{1} \mathrm{~d} y \int_{y}^{x} \mathcal{D}_{s} G_{x ; s}^{x ; y} M_{s ; x}^{y ; x}\right\}
\end{aligned}
$$

Let us now work out two examples :

- $\delta / \delta M_{t ; t}^{y ; x}, t<x<y$.

$$
G_{t_{i} t}^{x ; y} \mathrm{~d} x \mathrm{~d} y \mathrm{~d} t=\sum_{k} \frac{\delta \mathcal{L}}{\delta K_{k}^{y ; x}} \frac{\delta K_{k}^{y ; x}}{\delta M_{t_{i} t}^{y ; x}}
$$

where $\delta \mathcal{L} / \delta K_{k}^{y ; x}$ is given by the bracketed term in (4.2) and from (2.6.a), $k<x<y$, one gets

$$
\frac{\delta K_{k}^{y ; x}}{\delta M_{t ; t}^{y ; x}}=-\int_{k}^{x} \mathcal{D}_{z} \delta(t-z) \mathrm{d} t
$$

Hence

$$
G_{t ; t}^{x ; y}=-\sum_{k} \frac{\mu(k)}{n} F_{k}^{x ; y}[\Theta(t-k)+k \delta(t-k)]=\int_{0}^{t} D_{k} F_{k}^{x ; y}
$$

retrieving (2.1.c)

- $\delta / \delta M_{1 ; x}^{y ; x}, x<y$.

The chain rule gives

$$
\frac{\delta K_{k}^{y ; x}}{\delta M_{1 ; x}^{y ; x}}=+4
$$

thus leading to

$$
G_{x ; 1}^{x ; y} \mathrm{~d} x \mathrm{~d} y=\sum_{k} \frac{\mu(k)}{n} F_{k}^{x ; y} \Delta_{x}^{(k)} \Delta_{y}^{(k)}
$$


with $\Delta_{t}^{(k)}$ as in (2.17), $\mu(k)$ as in (10); after a partial integration one gets

$$
G_{x ; 1}^{x ; y}=-\left(\int_{0}^{x}+\frac{1}{2} \int_{x}^{y}+\frac{1}{4} \int_{y}^{1}\right) \frac{\mathrm{d} k}{k} \frac{\partial}{\partial k} F_{k}^{x ; y}+\frac{1}{4} F_{1}^{x ; y}
$$

identical to (2.1.a) with $t \equiv 1$.

\section{The bare case.}

Let us illustrate the above by considering now the simplest case i.e. the bare mass operator ${ }^{0} M^{\alpha \beta ; \gamma \delta}$ We have obtained it in (1.15), (2.8) (from the Parisi Lagrangean after shifting the field $\varphi_{\alpha \beta}=q_{\alpha \beta}+\delta \varphi_{\alpha \beta}$ and retaining the quadratic terms in $\delta \varphi$ as in (3)) with the $k$ independent kernel

$$
{ }^{0} K_{k}^{x ; y}=-4 w q(\min x, y)
$$

To be slightly more general let us add to the Parisi Lagrangean the quartic coupling term $\frac{v}{8} \operatorname{tr} \varphi^{4}$. One can calculate the new mass operator (which now contains a non vanishing ${ }^{0} M^{\alpha \beta ; \gamma \delta}$ component in contrast to the pure $\frac{w}{6} \operatorname{tr} \varphi^{3}$ Lagrangean) and its kernel:

$$
{ }^{0} K_{k}^{x ; y}= \begin{cases}A_{k}^{-}(\min x, y) & \min (x, y)<k \\ A_{k}^{+}(\min x, y) & \min (x, y)>k\end{cases}
$$

with

$$
\begin{aligned}
& \frac{1}{4} A_{k}^{-}(x)=-w q(x)-v\left(q^{2}\right)(x)+v q(x)\left[k q(k)+\int_{k}^{1} \mathrm{~d} t q(t)\right] \\
& \frac{1}{4} A_{k}^{+}(x)=-w q(x)-v\left(q^{2}\right)(x)+\frac{v}{2}\left[x q^{2}(x)+k q^{2}(x)+2 q(x) \int_{x}^{1} \mathrm{~d} t q(t)+\int_{k}^{x} \mathrm{~d} t q^{2}(t)\right]
\end{aligned}
$$

which is now $k$ dependent, but preserves the crucial property of only depending on the single running variable $\min (x, y)$.

For completeness we also give the replicon kernel

$$
\begin{aligned}
K_{k ; \ell}^{x ; x} & =p^{2}-2 \tau-2 u q^{2}(x)+E^{0}(k)+E^{0}(\ell) \\
& +v\left[2 \int_{0}^{1} \mathrm{~d} s q^{2}(s)-\mathcal{T}(k)-\mathcal{T}(\ell)-\frac{E^{0}(k)}{w} \frac{E^{0}(\ell)}{w}\right]
\end{aligned}
$$

with $E^{0}$ as in (1.17) and

$$
\begin{aligned}
& T(k)=k T(k)+\int_{k}^{1} \mathrm{~d} s T(s) \\
& T(k)=\int_{0}^{k} \mathrm{~d} s q^{2}(s)+k q^{2}(k)+2 q(k) \int_{k}^{1} \mathrm{~d} s q(s)
\end{aligned}
$$

Let us now rewrite the eigenvalue equation (2.16) and the integral equation $(4.4,5)$. Remember we have defined (1.16)

$$
{ }^{0} K_{x: k}^{x ; x}(p) \equiv p^{2}+\lambda_{p}^{0}(x ; x, k) \equiv \Lambda_{k}^{0}(x)
$$


then we get

$$
\Lambda_{k}^{0}(x) \varphi_{k}(x)-\int_{0}^{1} A_{k}(\min x, t) \frac{\Delta_{t}^{(k)}}{2 \Lambda_{k}^{0}(t)} \varphi_{k}(t)=\lambda_{k}^{0} \varphi_{k}(x)
$$

and

$$
\hat{F}_{k}^{x ; y}=A_{k}(\min x, y)+\int_{0}^{1} A_{k}(\min x, t) \frac{\Delta_{t}^{(k)}}{2 \Lambda_{k}^{0}(t)} \hat{F}_{k}^{t ; y}
$$

The propagator kernel $\hat{F}$, then satisfies the Sturm Liouville equation

$$
\frac{\mathrm{d}}{\mathrm{d} x} \frac{1}{\dot{A}_{k}(x)} \frac{\mathrm{d}}{\mathrm{d} x} \hat{F}_{k}^{x ; y}=-\frac{\hat{F}_{k}^{x ; y}}{2 \Lambda_{k}^{0}(x)}
$$

where $A_{k}(x)$ and $\Lambda_{k}^{0}(x)$ have distinct analytical expressions for $x \lessgtr k$ (as in (5.2) for $A$, and $\Lambda_{k}(x)=\Lambda_{x}(x)$ for $\left.k<x\right)$.

To solve for $(5.6,7)$ it is useful to come back to the evaluation of the determinant of eigenvalues as in (3.4). Instead of using the formula det $=\exp \operatorname{tr} \ln$, let us use the alternative determinant expansion for $\boldsymbol{\Delta}_{k}=\operatorname{det}\left\{\mathbb{1}-N_{x, y}\right\}$

$$
\Delta_{k}=1-\sum_{1} N_{11}+\sum_{1<2}\left|\begin{array}{ll}
N_{11} & N_{12} \\
N_{21} & N_{22}
\end{array}\right|-\sum_{1<2<3}\left|\begin{array}{lll}
N_{11} & N_{12} & N_{13} \\
N_{21} & N_{22} & N_{23} \\
N_{31} & N_{32} & N_{33}
\end{array}\right|+\cdot
$$

and now, because the kernel $K^{x ; y}$ only depends upon $\min (x ; y)$ we have

$$
N_{x ; y}=K_{k}^{x ; y} \frac{\Delta_{y}^{(k)}}{2 \Lambda_{k}(y)}
$$

$1 \mathrm{e}$.

$$
N_{x ; y}=A_{k}(\min x, y) \frac{\Delta_{y}^{(k)}}{2 \Lambda_{k}(y)}
$$

Now the last written term of the expansion (5.9) is

$$
-\sum_{1<2<3}\left|\begin{array}{lll}
A(1) \frac{\Delta_{1}}{2 \Lambda^{0}(1)} & A(1) \frac{\Delta_{2}}{2 \Lambda^{0}(2)} & A(1) \frac{\Delta_{3}}{2 \Lambda^{0}(3)} \\
A(1) \frac{\Delta_{1}}{2 \Lambda^{0}(1)} & A(2) \frac{\Delta_{2}}{2 \Lambda^{0}(2)} & A(2) \frac{\Delta_{3}}{2 \Lambda^{0}(3)} \\
A(1) \frac{\Delta_{1}}{2 \Lambda^{0}(1)} & A(2) \frac{\Delta_{2}}{2 \Lambda^{0}(2)} & A(3) \frac{\Delta_{3}}{2 \Lambda^{0}(3)}
\end{array}\right|
$$

where we have dropped the $k$ index in $A_{k}, \Delta^{(k)}$ and $\Lambda_{k}^{0}$. From (5.12) and alike higher order terms, one easily generates

$$
\begin{aligned}
\Delta_{k} & =1-\sum_{1} \frac{A_{1} \Delta_{1}}{2 \Lambda_{1}}+\sum_{1<2} \frac{A_{1} \Delta_{1}}{2 \Lambda_{1}}\left(A_{2}-A_{1}\right) \frac{\Delta_{2}}{2 \Lambda_{2}} \\
& -\sum_{1<2<3} \frac{A_{1} \Delta_{1}}{2 \Lambda_{1}}\left(A_{2}-A_{1}\right) \frac{\Delta_{2}}{2 \Lambda_{2}}\left(A_{3}-A_{2}\right) \frac{\Delta_{3}}{2 \Lambda_{3}}+.
\end{aligned}
$$


Let us now introduce the auxiliary functions $\phi^{ \pm}\left({ }^{5}\right)$

$$
\begin{aligned}
4 \phi^{+}(u) & =-A_{u}-\sum_{1<u} \frac{A_{1} \Delta_{1}}{2 \Lambda_{1}}\left(A_{u}-A_{1}\right)+\ldots \\
\phi^{-}(v) & =1-\sum_{v<1}\left(A_{1}-A_{v}\right) \frac{\Delta_{1}}{2 \Lambda_{1}}+\ldots
\end{aligned}
$$

which satisfy the integral equations

$$
\begin{aligned}
4 \phi^{+}(u) & =-A_{u}-\int_{0}^{u}\left(A_{u}-A_{t}\right) \frac{\Delta_{t}}{2 \Lambda_{t}} 4 \phi^{+}(t) \\
\phi^{-}(v) & =1-\int_{v}^{1}\left(A_{v}-A_{t}\right) \frac{\Delta_{t}}{2 \Lambda_{t}} \phi^{-}(t)
\end{aligned}
$$

They also satisfy the same Sturm Liouville equation as in (5.8)

$$
\frac{\mathrm{d}}{\mathrm{d} x} \frac{1}{\dot{A}_{k}(x)} \frac{\mathrm{d}}{\mathrm{d} x} \phi_{k}^{ \pm}(x)=-\frac{\phi_{k}^{ \pm}(x)}{2 \Lambda_{k}(x)}
$$

where we have now restituted the $k$ index.

Note that $\Delta_{k}$ is the Wronskian

$$
\boldsymbol{\Delta}_{k}=\left(\dot{\phi}_{k}^{+} \phi_{k}^{-}-\phi_{k}^{+} \dot{\phi}_{k}^{-}\right) / \dot{A}_{k}
$$

and

$$
\Delta_{k}=1+\int_{0}^{1} \frac{\Delta_{t}^{(k)}}{2 \Lambda_{k}(t)} 4 \phi_{k}^{+}(t)=1-\int_{0}^{1} A_{k}(t) \frac{\Delta_{t}^{(k)}}{2 \Lambda_{k}(t)} \phi_{k}^{-}(t)
$$

It is then a simple matter to verify that

$$
F_{k}^{x ; y}=\frac{1}{\Lambda_{k}(x)} \frac{4 \phi_{k}^{+}(\min x, y) \phi_{k}^{-}(\max x, y)}{\Delta_{k}} \frac{1}{\Lambda_{k}(y)}
$$

which also follows from standard Green's function theory (Morse and Feshbach [16]).

For completeness, we may now recover ${ }_{A} G_{1 ; 1}^{x ; x}$ by differentiating with respect to ${ }^{0} M^{\alpha \beta ; \alpha \beta}(p)$ as we did to obtain (1.19). From

$$
-\beta_{\mathrm{A}} f_{0} n=-\frac{1}{2} \sum_{k} \mu(k) \ln \Delta_{k}
$$

one gets

$$
\left.{ }^{0} G^{\alpha \beta ; \alpha \beta}\right|_{(\alpha \cap \beta)=x}=\mathrm{d} x_{\mathrm{A}} G_{1 ; 1}^{x ; x}=\sum_{k} \frac{\mu(k)}{n} \frac{1}{\Delta_{k}} \frac{\partial}{\partial^{0} M^{x ; x}(p)} \Delta_{k} .
$$

Performing the differentiation on the expanded form (5.13) of $\Delta_{k}$ one immediately recognizes, comparing with $(5.14,15)$, the combination

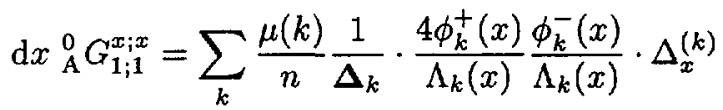

$\left({ }^{5}\right)$ It would have been nicer to choose $4 \phi^{+}$instead of $\phi^{+}$as an auxiliary function. We have kept the old notation of [I,II] to avold confusion. 
i.e. after partial integration

$$
{ }_{\mathrm{A}}^{0} G_{1 ; 1}^{x ; x}=-\left(\int_{0}^{x}+\frac{1}{2} \int_{x}^{1}\right) D_{k} F_{k}^{x ; x}+\frac{1}{2} F_{1}^{x ; x}
$$

in agreement with (2.2) and (2.1.a).

Originally (5.13-21) were written for the Parisi Lagrangean with $A(x)=-4 q(\min x, y)$ and $\phi^{ \pm}$explicitly constructed in [I] (with the occurrence of Gegenbauer functions). At that time the central role of kernels was not understood, as a result it was believed that one could not explicitly write the propagators when a $\frac{v}{8} \operatorname{tr} \varphi^{4}$ term was added to the Parisi Lagrangean.

What we have done so far can be repeated when keeping the full quartic invariants of the theory. The extra invariants involved produce separable terms adding to (5.1). This more general case can also be resolved for $F_{k}^{x ; y}$ through a non trivial calculation. Details will be published separately.

\section{Introducing the magnetic field.}

Let us return to the way we recovered the longitudinal anomalous propagator. For example one had, for $x<y$

$$
\mathrm{d} x \mathrm{~d} y G_{x ; 1}^{x ; y}=\sum_{k} \frac{\mu(k)}{n} F_{k}^{x ; y} \Delta_{x}^{(k)} \Delta_{y}^{(k)}
$$

which could by converted into (4.12) via a partial integration. This operation amounts to the rewriting, in the discrete representation,

$$
\frac{1}{p_{0}} \phi_{0}^{r ; s}+\sum_{k=1}\left(\frac{1}{p_{k}}-\frac{1}{p_{k-1}}\right) \phi_{k}^{r ; s}=\frac{1}{p_{0}}\left(\phi_{0}^{r ; s}-\phi_{1}^{r ; s}\right)+\sum_{k=1} \frac{1}{p_{k}}\left(\phi_{k}^{r ; s}-\phi_{k+1}^{r ; s}\right)
$$

where we forget about the effect of $\Delta^{(k)}$ (we assume that $r \equiv x(R+1), s \equiv y(R+1)$ are far from the origin). The right hand side then becomes $-(\mathrm{d} k / k) \partial / \partial k \phi_{k}^{x ; y}$ in the continuum leading to (4.12). However, returning to the definition of $F$ as in (4.2-4), it is easily seen that $F$ has the discontinuity

$$
F_{1}^{r ; s}-F_{0}^{r ; s}=\delta F^{r ; s} p_{0}
$$

Indeed, this is due to the discontinuity of $\Delta_{r}^{(k)}$ itself for $r=0$. This is obvious in the definition of the discrete ultrametric weight $\Delta^{(k)}$ (the continuous version was given in (2.17))

$$
\begin{array}{rlrl} 
& \frac{1}{2}\left(p_{r+1}-p_{r}\right) & r<k-1 \\
\Delta_{r}^{(k)}= & \frac{1}{2}\left(p_{k}-p_{k-1}\right)+\frac{1}{2} p_{k} & r=k-1 \\
& \left(p_{r+1}-p_{r}\right) & r>k-1
\end{array}
$$

i.e.

$$
\Delta_{r=0}^{(1)}-\Delta_{r=0}^{(0)}=\frac{p_{0}}{2}
$$

which induces a discontinuity like (6.2) via (4.2) or (4.4). It turns out that neither $K_{k}$ nor $K_{k, \ell}$ possess such a discontinuity (this can be checked at the bare level; see below for more on that). We can then work out $\delta F^{r ; s}$ using the generating functional method. First we consider the 
$\log$ term (there is no $\Delta^{(k)}$ weight in the replicon component so we restrict ourselves to $\mathcal{L}_{\mathrm{LA}}$ in (3.5), the longitudinal anomalous one) and write the term $\delta \mathcal{L}_{\mathrm{LA}}$ generated by the discontinuity (6.4)

$$
\delta \mathcal{L}_{\mathrm{LA}}=\frac{1}{p_{0}}\left\{\operatorname{tr} \ln \left\{\mathbb{1}-\frac{1}{\Lambda_{0}(r)} K_{0}^{r ; s} \frac{\Delta_{s}^{(0)}}{2}\right\}-\operatorname{tr} \ln \left\{\mathbb{1}-\frac{1}{\Lambda_{1}(r)} K_{1}^{r ; s} \frac{1}{2}\left(\Delta_{s}^{(0)}+\frac{p_{0}}{2}\right)\right\}\right\}
$$

Here $\Lambda_{1} \sim \Lambda_{0}, K_{1} \sim K_{0}$ (within $1 / R$ corrections), hence

$$
\delta \mathcal{L}_{\mathrm{LA}}=\frac{1}{2} \sum_{r}\left[\frac{1}{\mathbb{1}-\frac{1}{\Lambda_{0}} K_{0} \frac{\Delta^{(0)}}{2}}\right]_{0, r} \frac{1}{\Lambda_{0}(r)} K_{0}^{r ; 0} \frac{1}{2}
$$

$1 \mathrm{e}$.

$$
\delta \mathcal{L}_{\mathrm{LA}}=\frac{1}{2} \cdot \frac{1}{2 \Lambda_{0}(0)}\left\{\sum_{r} K_{0}^{0 ; r} \frac{\Delta_{r}^{(0)}}{2 \Lambda_{0}(r)} K_{0}^{r ; 0}+\sum_{r ; s} K_{0}^{0 ; r} \frac{\Delta_{r}^{(0)}}{2 \Lambda_{0}(r)} K_{0}^{r ; s} \frac{\Delta_{s}^{(0)}}{2 \Lambda_{0}(s)} K^{s ; 0}+. .\right\} .
$$

To complete the new functional we need write now the term in $\delta \operatorname{tr} \overline{\bar{G}} \overline{\bar{M}}$ supplementing (3.9):

$$
\delta \operatorname{tr} \overline{\bar{G}} \overline{\bar{M}}=-\sum_{r ; s} \delta F^{r ; s} \frac{\Delta_{s}^{(0)}}{2} K_{0}^{s ; r} \frac{\Delta_{r}^{(0)}}{2}-\sum_{r} \delta F^{r ; r} K_{0}^{r ; r} \frac{\Delta_{r}^{(0)}}{2}
$$

The new functional $n(\mathcal{F}+\delta \mathcal{F})$ can now be written with

$$
n \delta \mathcal{F}=\delta \mathcal{L}_{\mathrm{LA}}-\delta \operatorname{tr} \overline{\bar{G}} \overline{\bar{M}}
$$

(we now return to the continuous notation $x, y, t, \ldots$ ).

Stationarity with respect to $K_{0}^{y ; x}$ leads to $(x, y \neq 0)$

$$
\begin{aligned}
\delta F^{x ; y} \frac{\Delta_{x}^{(0)}}{2} \frac{\Delta_{y}^{(0)}}{2} & =\sum_{t} \frac{1}{2}\left[\frac{1}{\mathbb{1}-\frac{1}{\Lambda_{0}} K_{0} \frac{\Delta^{(0)}}{2}}\right]_{0 ; y} \frac{\Delta_{x}^{(0)}}{2 \Lambda_{0}(y)}\left[\frac{1}{1-\frac{1}{\Lambda_{0}} K_{0} \frac{\Delta^{(0)}}{2}}\right]_{x ; t} \frac{1}{2 \Lambda_{0}(t)} K_{0}^{t ; 0} \\
& =\frac{1}{2 \Lambda_{0}(0)} \frac{\Delta_{x}^{(0)}}{2 \Lambda_{0}(x)} \frac{\Delta_{y}^{(0)}}{2 \Lambda_{0}(y)}\left\{K_{0}^{x ; 0} K_{0}^{0 ; y}+K_{0}^{x ; 0} \sum_{t} K_{0}^{0 ; t} \frac{\Delta_{t}^{(0)}}{2 \Lambda_{0}(t)} K_{0}^{t ; y}\right. \\
& \left.+\sum_{t} K_{0}^{x ; t} \frac{\Delta_{t}^{(0)}}{2 \Lambda_{0}(t)} K_{0}^{t ; 0} K_{0}^{0 ; y}+\ldots\right\}
\end{aligned}
$$

We thus have the final result

$$
\delta F^{x ; y}=\frac{1}{2} \Lambda_{0}(0) F_{0}^{0 ; y} F_{0}^{x ; 0}
$$

since with (4.2)

$$
-F^{x ; 0}=\frac{1}{\Lambda_{0}(x)}\left\{K_{0}^{x ; 0}+\sum_{t} K_{0}^{x ; t} \frac{\Delta_{t}^{(0)}}{2 \Lambda_{0}(t)} K_{0}^{t ; 0}+. .\right\} \frac{1}{\Lambda_{0}(0)} .
$$


In the bare limit we have

$$
\delta F^{x ; y}=\frac{8}{\Lambda_{0}(0)}\left(\frac{\phi_{0}^{+}(0)}{\Delta_{0}}\right)^{2} \frac{\phi_{0}^{-}(x)}{\Lambda_{0}(x)} \frac{\phi_{0}^{-}(y)}{\Lambda_{0}(y)}
$$

Note that $\phi_{0}^{+}(0)$ vanishes in zero field, hence there is no "surface" term in that case. In field the change is summarized by the occurrence of the same increment $\delta F^{x ; y}$ for each sector of $G^{x ; y}$ i.e. $\delta F^{x ; y}$ is to be added to each right hand side of $(2.1 \mathrm{a}, \mathrm{b}, \mathrm{c})$ and $(2.2)$. Of course $F_{k}^{x ; y}$ is itself field dependent (so are, in the bare case, $\phi_{k}^{ \pm}, \Delta_{k}$ ). Note also that all integrations containing $\partial / \partial k$, or $\partial / \partial t, \partial / \partial z$ vanish in the interval $\left(0, x_{0}\right)$ since $q(x)=q_{0}$ for $x \leq x_{0}$; in the replicon sector this is the only way the magnetic field is felt.

Thus, for example in replacement of $(2.1 \mathrm{c})$, one should now $\operatorname{read}\left({ }^{6}\right)$

$$
\begin{array}{ll}
x_{0}<t<x<y & G_{t ; t}^{x ; y}=\delta F^{x ; y}+\int_{x_{0}}^{t} D_{k} F_{k}^{x ; y} \\
t<x_{0}<x<y & G_{t ; t}^{x ; y}=\delta F^{x ; y}
\end{array}
$$

\section{Status of perturbation theory and conclusion.}

Via perturbation one has access to the loop expansion of $M^{\alpha \beta ; \gamma \delta}$ i.e. to the components $M^{x ; y}$ and ${ }_{\mathrm{A}} M^{x ; x},{ }_{\mathrm{R}} M^{x_{i} x}$. With the components one can reconstruct the kernels $K_{k}^{x ; y}, K_{k ; \ell}^{x ; x}$ which are our only access to the full propagators. In the replicon case one possesses an explicit expression of ${ }_{\mathrm{R}} G^{x ; x}$ versus $K_{k ; \ell}^{x ; x}$. In the anomalous case one has to solve the Dyson's equation (4.3) or (4.4). Despite the simplifications brought up by the above analysis, even the one-loop calculation of the mass operator $M^{\alpha \beta ; \gamma \delta}$ is not yet an easy task to perform, and it would certainly be very convenient, for further analysis, to have it available. For example, one can write the pure longitudinal-anomalous contribution to $M_{k}^{x ; y}$ as

$$
\begin{aligned}
M_{k}^{x ; y} & =\sum\left\{{ }_{1} W_{k}^{x ; y}\left(u, v ; k_{1}, k_{2}\right)\left(F_{k_{1}}^{x ; y} F_{k_{2}}^{u ; v}+F_{k_{1}}^{x ; v} F_{k_{2}}^{y ; u}\right)\right. \\
& +{ }_{2} W_{k}^{x ; y}\left(u, v ; k_{1}, k_{2}\right) F_{k_{1}}^{u ; y} F_{k_{2}}^{u ; v} \\
& +{ }_{3} W_{k}^{x ; y}\left(u, v ; k_{1}, k_{2}\right) F_{k_{1}}^{x ; v} F_{k_{2}}^{u ; v} \\
& \left.+{ }_{4} W_{k}^{x ; y}\left(u, v ; k_{1}, k_{2}\right) F_{k_{1}}^{u ; v} F_{k_{2}}^{u ; v}\right\}
\end{aligned}
$$

and alike for the replicon or mixed contributions. Here $\sum$ is for integration over repeated variables $u, v, k_{1}, k_{2}$. A knowledge of these weights, which are to be simple functions of the arguments and straightforward (but lengthy) to compute, would certainly be desirable.

To conclude, we have provided the full inversion of the mass operator matrix, at the price, in the longitudinal-anomalous sector, of solving a Dyson equation with a non diagonal kernel. Still a very difficult task in the considered case of the standard spin glass. However in systems that are expected to be simpler like manifolds in random media (Mézard and Parisi [17] and Goldschmidt [18]) the Dyson equation (for pairs of propagators) becomes soluble and the inversion can be carried out to its end, a result that will be published separately. In the bare case we have given the full solution in a field to the inversion problem, including when one

$\left({ }^{6}\right)$ In previous work $[I, I I]$, the surface term $\delta F$ was overlooked. 
keeps quartic invariants in the Lagrangean. Again a problem that was believed so far to be beyond the possibility of a complete solution.

These two examples demonstrate that the reduction of the inversion of an ultrametric matrix to the resolution of a (formally simple) Dyson's equation has enlarged the field of accessible problems.

\section{Acknowledgements.}

One of the authors (C.D) would like to thank M. Mézard and G. Parisi for useful and stimulating discussions.

\section{Appendix}

\section{Propagators.}

We first write the propagators $G$ in a suggestive form i.e. as a summation of the kernel $F$ over eigenvalue indices ( $k$, or $k$ and $\ell$ ) weighted by multiplicities and ultrametric factors (aequations). Via partial integration, we then exhibit them in fully explicit form (b-equations). We end up with remarks on ultrametric weights, on the passage from $a$ to $b$ and simplified expression for the propagators.

Note that everything written here for the pair $G, F$ stands also for $M, K$.

(i) REPLICON SECTOR

First propagator:

$$
-n \frac{\mathrm{d} x}{2}{ }_{\mathrm{R}} G_{1 ; 1}^{x ; x}=\sum_{k, \ell} \mu_{\mathrm{reg}}(x ; k, \ell) F_{k ; \ell}^{x ; x} U_{k}(x)
$$

1 e. after partial integration

$$
\begin{aligned}
\mathrm{R}_{1 ; 1} G_{1 ; 1}^{x ; x} & =\int_{x}^{x_{1}} \frac{\mathrm{d} k}{k} \frac{\partial}{\partial k} \int_{x}^{x_{1}} \frac{\mathrm{d} \ell}{\ell} \frac{\partial}{\partial \ell} F_{k ; \ell}^{x ; x} \\
& -\int_{x}^{x_{1}}\left(\frac{\mathrm{d} k}{k} \frac{\partial}{\partial k} F_{k ; x_{1}}^{x ; x}+\frac{\mathrm{d} \ell}{\ell} \frac{\partial}{\partial \ell} F_{x_{1} ; \ell}^{x ; x}\right) \\
& +F_{x_{1} ; x_{1}}^{x ; x}
\end{aligned}
$$

Second propagator:

$$
-n \frac{\mathrm{d} x}{2}{ }_{\mathrm{R}} G_{1 ; z}^{x ; x}=\sum_{k, \ell} \mu_{\mathrm{reg}}(x ; k, \ell) F_{k ; \ell}^{x ; x} V_{k}(z)
$$

here $1+V_{k}(z)=2 U_{k}(z)$ and $U_{k}(z)$ defined in $(2.17) ; V_{k}(z)$ can also be generated as $\delta E(k) / \delta w q(z)$ with $E^{0}(k)$ as in $(1.17)$.

$$
{ }_{\mathrm{R}} G_{1 ; z}^{x ; x}=\int_{x}^{x_{1}} \frac{\mathrm{d} k}{k} \frac{\partial}{\partial k} \int_{x}^{z} \frac{\mathrm{d} \ell}{\ell} \frac{\partial}{\partial \ell} F_{k ; \ell}^{x ; x}-\int_{x}^{z} \frac{\mathrm{d} k}{k} \frac{\partial}{\partial k} F_{k ; x_{1}}^{x ; x}
$$


Third propagator:

$$
\begin{aligned}
-n \frac{\mathrm{d} x}{2}{ }_{\mathrm{R}} G_{z_{1} ; z_{2}}^{x ; x} & =\sum_{k, \ell} \mu_{\mathrm{reg}}(x ; k, \ell) F_{k ; \ell}^{x ; x} V_{k}\left(z_{1}\right) V_{k}\left(z_{2}\right) \\
{ }_{\mathrm{R}} G_{z_{1} ; z_{2}}^{x ; x} & =\int_{x}^{z_{1}} \frac{\mathrm{d} k}{k} \frac{\partial}{\partial k} \int_{x}^{z_{2}} \frac{\mathrm{d} \ell}{\ell} F_{k ; \ell}^{x ; x}
\end{aligned}
$$

The discontinuity occurring when two close replicas come to coincide, manifests itself here by the disappearance of a $V$ weight: $V_{k}(z)=1$ as $z \equiv 1$ (see below).

(ii) LONGITUDINAL-ANOMALOUS SECTOR

$$
x<y \quad G_{. ; t}^{x ; y}=\sum_{k} n^{-1} \mu(k) F_{k}^{x ; y} U_{k}(x) U_{k}(y) V_{k}(t)
$$

where the unspecified lower index (replaced by a dot) can be either $x$ or $t, x \leq t$. The explicit form, for various respective order of $x, y, t$ becomes

$$
\begin{aligned}
& x<y<t \quad G_{x ; t}^{x ; y}=\delta F^{x ; y}-\left(\int_{x_{0}}^{x}+\frac{1}{2} \int_{x}^{y}+\frac{1}{4} \int_{y}^{t}\right) \frac{\mathrm{d} k}{k} \frac{\partial}{\partial k} F_{k}^{x ; y} \\
& x<t<y \quad G_{x ; t}^{x ; y}=G_{t \cdot t}^{x ; y}=\delta F^{x ; y}-\left(\int_{x_{0}}^{x}+\frac{1}{2} \int_{x}^{t}\right) \frac{\mathrm{d} k}{k} \frac{\partial}{\partial k} F_{k}^{x ; y} \\
& t<x<y \quad G_{t ; t}^{x ; y}=\delta F^{x ; y}-\int_{x_{0}}^{t} \frac{\mathrm{d} k}{k} \frac{\partial}{\partial k} F_{k}^{x ; y}
\end{aligned}
$$

and for a coinciding pair

$$
\begin{aligned}
x<y \quad G_{x ; 1}^{x ; y} & =\sum_{k} n^{-1} \mu(k) F_{k}^{x ; y} U_{k}(x) U_{k}(y) \\
G_{x ; 1}^{x ; y} & =\delta F^{x ; y}-\left(\int_{x_{0}}^{x}+\frac{1}{2} \int_{x}^{y}+\frac{1}{4} \int_{y}^{x_{1}}\right) \frac{\mathrm{d} k}{k} \frac{\partial}{\partial k} F_{k}^{x ; y}+\frac{1}{4} F_{1}^{x ; y}
\end{aligned}
$$

Other components are obtained for $y \longrightarrow x$ and use of $(2.2)$.

(iii) REMARKS ON THE WEIGHTS

To the lower indices is attached the weight

$$
V_{k}(z)=\Theta(z-k)+k \delta(z-k)
$$

To the upper indices in the longitudinal-anomalous sector we have the weight (2.17),

$$
U_{k}(x)=\Theta(x-k)+\frac{k}{2} \delta(x-k)+\frac{1}{2} \Theta(k-x)
$$


In the replicon sector the weight attached to the upper index, $W_{k}(x)$ is carried by the multiplicity (12)

$$
\begin{aligned}
\mu_{\text {reg }}(x ; k, \ell) & =\left(-\frac{n}{2} \mathrm{~d} x\right) \frac{\mathrm{d} k}{k^{2}} \frac{\mathrm{d} \ell}{\ell^{2}} W_{k}(x) W_{\ell}(x) \\
W_{k}(x) & =\Theta(k-x)-k \delta(k-x)
\end{aligned}
$$

Note that if the domain of integration of $k$ (or $\ell$ ) is restricted to $\left(0 ; 1_{-}\right)$, then $V_{k}(z)=1$ when $z \equiv 1$. Thus we have a single expression for all replicon propagators which writes $(x \leq z \leq 1)$

$$
{ }_{\mathrm{R}} G_{z_{1} ; z_{2}}^{x ; x}=\sum_{k, \ell} \frac{\mu(k)}{n} \frac{\mu(\ell)}{n} F_{k ; \ell}^{x ; x} W_{k}(x) W_{\ell}(x) V_{k}\left(z_{1}\right) V_{\ell}\left(z_{2}\right)
$$

and a single expression for the longitudinal-anomalous propagators

$$
G_{; z}^{x ; y}=\sum_{k} \frac{\mu(k)}{n} F_{k}^{x ; y} U_{k}(x) U_{k}(y) V_{k}(z)
$$

supplemented by eq.(2.2).

Note finally that under partial integration with $\mu(k) / n \equiv-\mathrm{d} k / k^{2}, V_{k}(z)$ and $W_{k}(x)$ lead respectively to $-\int^{z} \frac{\mathrm{d} k}{k} \frac{\partial}{\partial k}$ and $-\int_{x} \frac{\mathrm{d} k}{k} \frac{\partial}{\partial k}$. Since

$$
U_{k}(x)=\frac{1}{2} W_{k}(x)+V_{k}(x)
$$

$U_{k}(x)$ leads to $-\left(\int^{x}+\frac{1}{2} \int_{x}\right) \frac{\mathrm{d} k}{k} \frac{\partial}{\partial k}$.

This shows how one goes from the a-equations to the b-explicit forms.

\section{References}

[1] Harris A.B., Lubensky T.C. and Chen J.H., Phys. Rev. Lett. 36 (1976) 415.

[2] Elderfield D. and Mc Kane, Phys. Rev. B18 (1978) 3730.

[3] Green J.E., J. Phys. A18 (1985) L43.

[4] De Dominicis C. and Kondor I., Phys. Rev. B27 (1983) 606.

[5] Parısi G., Phys. Lett. 73A (1979) 154; J. Phys. A13 (1980) L115, 1101, 1887.

[6] De Dominicis C. and Kondor I., Lecture Notes in Physics, Garrido ed. (Springer Verlag) 216 (1984) 91.

[7] De Dominicıs C. and Kondor I., J. Phys. Lett. France 46 (1985) L1037.

[8] Kondor I. and De Dominicis C., Europhys. Lett. 2 (1986) 617.

[9] Sompolinsky H. and Zippelius A., Phys. Rev. Lett. 50 (1983) 1297.

[10] Goltsev A.V., J. Phys. A16 (1983) 337; J. Phys. A17 (1984) 237.

[11] De Domınicıs C., Kondor I. and Temesvarı T., Int. J. Mod. Phys. B7 (1993) 986.

[12] de Almeida J.R.L. and Thouless D., J. Phys. A11 (1978) 983.

[13] Pytte G. and Rudnick J., Phys. Rev. B19 (1979) 3603.

[14] Bray A.J. and Moore M.A., J. Phys. C12 (1979) 79.

[15] Temesvari T., De Dominicis C. and Kondor I., to be published.

[16] Morse P.M. and Feschbach H., Methods of Theoretical Physıcs (Mc Graw-Hill, New York, 1953).

[17] Mézard M. and Parısı G., J. Phys. I France 2 (1992) 2231.

[18] Goldschmidt Y.Y., J. Phys. I France 4 (1994) 87. 